\title{
28 Research Square \\ Disseminated nocardiosis caused by Nocardia vulneris in a macroglobulinemia patient
}

Fu lan QIU

Zhiyi MA

Rongrong ZHONG

Hao nan HUANG

Yue Hua WANG

Hui Liu (D liuhui3156@163.com )

Dpartment of Clinical Laboratory, Fujian Longyan First Hospital/Longyan First Affiliated of Fujian Medical University

\section{Research Article}

Keywords: Disseminated nocardiosis, Nocardia vulneris, macroglobulinemia, fever, shortness of breath

Posted Date: April 23rd, 2021

DOI: https://doi.org/10.21203/rs.3.rs-413268/v1

License: (a) This work is licensed under a Creative Commons Attribution 4.0 International License. Read Full License 


\section{Abstract}

Headings Background: This is a case of a human disseminated nocardiosis caused by Nocardia vulneris which made the patient presenting with fever, cough, shortness of breath, muscle pain and multiple tubercle. Methods: Bacterial culture the blood, sputum, lung rinses and scalp pus samples of the patient, Nocardia vulneris was isolated and identified using the 16s ribosomal RNA gene sequence sequence data.

and determine the sensitivity of the isolated bacteria to antibiotics and analysis of the strain's antibiotic treatment. Results: The isolated was identified as Nocardia brasiliensis, which was resistant to ciprofloxacin, but susceptible to amikacin, gentamicin, tobramycin, linezolid, trimethoprimsulfamethoxazole, amoxicillin/clavulanic, moxifloxacin, ceftriaxone, cefotaxim, imipenem. The patient recovered and his condition remained stable by combinations with linezolid, amikacin and trimethoprimsulfamethoxazole. Conclusions: This is the first case report of disseminated nocardiosis caused by Nocardia vulneris, and the current case was treated successfully with linezolid, amikacin and trimethoprim-sulfamethoxazole. Clinicians should be aware of its diagnostic, and the MIC value of the drug sensitivity test should be concerned when there is a wide choice of medicines, for the disseminated cases which are diagnosed definitely should be treated with at least 12 months of antimicrobial therapy, bacteriological examination and antimicrobial susceptibility testing should be performed repeatedly.

\section{Introduction}

Nocardia species are soil saprophytes which are widespread in soil or water and always associated with pulmonary infection. They can cause serious human infections, especially in immunocompromised patients ${ }^{[1,2]}$. With the widly use of steroids, immunosuppressants, broad-spectrum antibiotics and the development of organ transplantation treatment, recent reports suggest that the incidence of nocardiosis is increasing. However, disseminated infections caused by Nocardia vulneris are rare and could with different clinical symptoms. In this study, we report an case of disseminated nocardiosis with the involvement of lung, brain, blood, skin caused by a rarely isolated species Nocardia vulneris in a macroglobulinemia patient.

\section{Case Presentation}

A 61-year-old man was diagnosed with macroglobulinemia for 4 years, he is undergoing a long-term use of prednisone acetate ( $15 \mathrm{mg} /$ day) and thalidomide ( $75 \mathrm{mg}$ qn). On December 5,2018 , the patient was sent to our hospital with a 1-month history of recurrent cough, expectoration, fever and shortness of breath, within the last week he had the symptoms of muscle pain in the limbs, pain in the joints, and headache. A physical examination revealed the following findings: Conscious, and systemic superficial lymph nodes were not touched. Double lung breath sound clear, no dry or wet rale. Rhythm of the heart is regular, no murmurs. Ventral soft, no tenderness and bounce painful, spine tenderness pain, without taps pain; Limb muscle tenderness, no rebound tenderness, each joint normal limbs form. No obvious pitting 
edema of double lower limbs, pathological character negative. Admission examination: body temperature $36.3^{\circ} \mathrm{C}$, respiration 20 times/min, pulse 92 times/min, pulmonary CT: multiple nodular shadows or lamellar shadows in the left lung, new appearance multiple lymph nodes in bilateral axilla and mediastinum, part of the lymph nodes swelling, as shown in Fig. 1. And Laboratory findings were as follows: Blood routine examination: WBC $3.35 \times 10^{9} / \mathrm{L}$, Neutrophile granulocyte $58.8 \%$, Hemoglobin $115 \mathrm{~g} / \mathrm{L}$, Blood platelet $193 \times 10^{9} / \mathrm{L}$, Urine routine: urine protein $1+$, urine sugar $1+$, urine protein 633.5 $\mathrm{mg} / \mathrm{L}$, immunoglobulin IgA $0.51 \mathrm{~g} / \mathrm{L}$, immunoglobulin IgG 4.54g/L, immunoglobulin IgG4.54g/L, immunoglobulin IgM6.15g/L, d-dimer $1.16 \mathrm{mg} / \mathrm{L}$, c-reactive protein $192.78 \mathrm{mg} / \mathrm{L}$, total protein $47.5 \mathrm{~g} / \mathrm{L}$, albumin $27.5 \mathrm{~g} / \mathrm{L}$, procalcin $0.54 \mathrm{ng} / \mathrm{ml}$. Levofloxacin was administered for 3 days, On December 7 , the patient began to have fever with a peak value of $39.5^{\circ} \mathrm{C}$. On December 8 , the patient still with high fever, and with the WBC $1.09 \times 10^{9} / \mathrm{L}$ and the absolute value of neutrophils was $0.41 \times 10^{9} / \mathrm{L}$. Then the patient discontinued treatment with steroids and immunosuppressants, and treated with recombinant human granulocyte stimulating factor $(90 \mathrm{ug} / \mathrm{day})$ to increase white blood cells, in addition, Meropenem ( $1 \mathrm{~g} /$ day) combinations with Doxycycline (100mg/day) to against infection. On December 9 , there were two nodules with a peanut size and a moderate hardness in the scalp, the right temporal area was accompanied by ulcer and obvious tenderness. The trunk and limbs were scattered with reddish plaque and nodules, with tenderness, and some skin lesions were the pustules on the basis of erythematous, as shown in Fig. 2. Gram positive bacilli (suspected Nocardia) were found in blood culture, further 16S rRNA gene identification confirmed the isolate as Nocardia vulneris. Based on the results of drug sensitivity, Trimethoprim-sulfamethoxazole (3g/day) treatment was instead of Doxycycline. Subsequently, the pus smear of sputum, lung washing fluid and scalp all revealed to be Nocardia, brain magnetic resonance imaging showed brain abscess as shown in Fig. 3 , and the patient's fever with a high peak of $40^{\circ} \mathrm{C}$. On December 12th, Trimethoprim-sulfamethoxazole ( $3 \mathrm{~g} /$ day) combined with ceftriaxone ( $2 \mathrm{~g} /$ day) treatment was started. However, the patient's symptoms did not abate. On December 14th, treatment was changed to SMZco combined with Linezolid, but on the December 15th, the patient's temperature haven't drop, and according to the drug susceptibility analysis, the MIC values of Linezolid, Amikacin and Trimethoprimsulfamethoxazole were far from the corresponding sensitive fold points, then the treatment was changed to the combination of Linezolid, Amikacin and Trimethoprim-sulfamethoxazole. On December 16th, there is a downturn in the highest temperature of the patient, the PCT and CRP significantly decreased. On December 24, the patient with normal temperature, no obvious chills, few occasional cough, shortness of breath phlegm can be produce. Skin rash, scalp abscess become narrowed, double lung breath sounds clear and a little wet rale, antibiotics was changed to Trimethoprim-sulfamethoxazole combined with Linezolid, the symptoms gradually improve, and many times of the microbial culture about the blood, phlegm and pus was negative, lung CT showed lesions absorption, the patient discharged from hospital on January 28 , and request the patient to continue long-term oral Trimethoprim-sulfamethoxazole combined Minocyline for 12 months. Follow-up lung CT and brain magnetic resonance (NMR) prompt lesions improved significantly after discharge.

\section{Microbiological Examination}




\subsection{Bacterial culture and identification}

A set of blood culture was sent for detection on December 7, and the blood culture was positive after culture for 35 hours, Gram positive long thin filamentous bacterial group was found in the smear, as shown in Fig. 4. White colonies of needle-size could be seen on the transferred blood plate after 24h. And large, dry, white colonies with obvious smell of dust could be seen after $72 \mathrm{~h}$, and which didn't embedded into the AGAR as shown in Fig. 5. Sputum and pus samples were inoculated into blood plate and cultured at $35^{\circ} \mathrm{C}$ for 48 hours, no growth of Nocardia was observed, however, fine colonies were observed after culture for 72 hours. Meanwhile, long filamentous gram positive bacterial group was found in sputum samples and scalp pus, and the weak acid-fast staining was positive, as shown in Fig. 6 . The colony was identified as Nocardia brasiliensis by mass spectrometry system Bruker (Bruker Company, Bruker MALDI Biotyper TOF, America ) and VITEK MS (BioMerieux Company, BioMerieux VITEK MS, China), and with the scores were 1.718 and 2.0 respectively. However, this isolates was identified as Nocardia vulneris by $16 \mathrm{~S}$ rRNA gene sequencing analysis (GenBank NR148265.1). The universal primers used for sequencing were (27F/1492R; 5'-AGAGTTTGATCCTGGCTCAG-3'/5'-CTACGGCTACCTTGTTACGA-3'), with the cycling conditions of initial denaturation $94^{\circ} \mathrm{C}$ for $5 \mathrm{~min}, 25$ cycles of denaturation $94^{\circ} \mathrm{C}$ for $30 \mathrm{Sec}$, annealing $57^{\circ} \mathrm{C}$ for $30 \mathrm{Sec}$, extension $72^{\circ} \mathrm{C}$ for $90 \mathrm{Sec}$, and a final extension $72^{\circ} \mathrm{C}$ for 5 min (RuiBo Biotechnology Company, Beijing, China); or used the primer (7F/1540R; 5'-CAGAGTTTGATCCTGGCT-3'/5'AGGAGGTGATCCAGCCGCA-3'), with the cycling conditions of initial denaturation $94^{\circ} \mathrm{C}$ for 4 min, 30 cycles of denaturation $94^{\circ} \mathrm{C}$ for $45 \mathrm{Sec}$, annealing $55^{\circ} \mathrm{C}$ for $45 \mathrm{Sec}$, extension $72^{\circ} \mathrm{C}$ for $1 \mathrm{~min}$, and a final extension $72^{\circ} \mathrm{C}$ for 10 min (Sangon Biotechnology Company, Shanghai, China).

\subsection{Drug Sensitivity Test}

The trace broth dilution method was used to determine the sensitivity of the strain to antibiotics, and CLSI M24 was used as the standard for determining drug sensitivity results, as shown in Table 1. 
Table 1

Susceptibility of Nocardia vulneris isolate to different antimicrobials.

\begin{tabular}{|lll|}
\hline Antimicrobials & MIC & Susceptibility \\
\hline Trimethoprim-Sulfamethoxazole & $<=0.5 / 9.5$ & $\mathrm{~S}$ \\
\hline Amoxycillin/clavulanicacid & $<=2 / 1$ & $\mathrm{~S}$ \\
\hline Amikacin & 2 & $\mathrm{~S}$ \\
\hline Gentamicin & $<=1$ & $\mathrm{~S}$ \\
\hline Tobramycin & $<=1$ & $\mathrm{~S}$ \\
\hline Imipenem & 4 & $\mathrm{~S}$ \\
\hline Linezolid & 1 & $\mathrm{~S}$ \\
\hline Ciprofloxacin & $>=8$ & $\mathrm{R}$ \\
\hline Moxifloxacin & 1 & $\mathrm{~S}$ \\
\hline Ceftriaxone & 8 & $\mathrm{~S}$ \\
\hline Cefotaxim & 8 & $\mathrm{~S}$ \\
\hline Cefepim & 16 & $\mathrm{I}$ \\
\hline Minocycline & 2 & $\mathrm{I}$ \\
\hline MIC, minimum inhibitory concentration; S, susceptible; I, intermediate; R, resistant.Susceptibility of the \\
\hline isolate to antimicrobials was defined according to the CLSI M24-A guidelines.
\end{tabular}

\section{Discussion}

As a soil saprophyte, Nocardia species invasion into human body through the respiratory tract, skin, or digestive tract. Nocardiosis usually prevalent in immunocompromised patients or affect the populations that secondary to other diseases, it is a life-threatening diseases to the infected patients ${ }^{[3]}$. Lung is the most commonly infective site of nocardiosis, accounting for about $70-80 \%[4,5]$, Gsenkirchen nocca, Nocardia brasiliensis, Ginea pig ncardia and Picrax ncardia are the main pathogenic bacteria of Nocardia species frequently isolated from humans. Disseminated infections caused by Nocardia usually affect immunocompromised patients ${ }^{[6]}$, and often originated from the lungs, then spread to the brain, the case fatality rate of disseminated infections is high ${ }^{[7]}$. However, there is also a report that a normal immune function individual with disseminated Nocardia species infections which originated from the lungs but spread to the brain and causing brain abscess ${ }^{[8]}$. But bloodstream infection is rare, and approximately $1.3-7.7 \%$ of nocardiosis may occur this case $\mathrm{e}^{[9,10]}$, and the mortality rate of the nocardia-bacteremia patients is high, may accounting for $50 \%{ }^{[11]}$. However, to the best of our knowledge, Nocardia vulneris is always manifest as skin and/or subcutaneous tissues infections, there are no reports on its role in disseminated infections, here, we present a cases of disseminated infection with Nocardia vulneris. 
Nocardia are not a part of the human normal flora, therefore it can be diagnosed as Nocardiosis when these organisms are isolated from sputum, alveolar lavage fluid, blood, pus, pleural fluid, subcutaneous tissues or other samples. In the case reported here, the patient was undergoing immunosuppressants and steroid treatment for gigantic globulin hematic disease for 4 years, he was admitted to the hospital suffering from a 1-month history of repeatedly cough associated with sputum, fever and shortness of breath, In addition, the patient with 1-week history of limbs muscle soreness, interphalangeal joint pain, and headache. A set of blood culture was started upon admission, and the aerobic blood culture was positive after culture for 35 hours, gram positive red filamentous branching bacterial group was found in the smear, although it is visible from the phlegm or alveolar lavage fluid direct smear specimens by the wright's dye and weak acid fast dye, but it is also easily be concealed by other bacterias which growth rapidly, so smear microscopic examination is very important in early diagnosis. In addition, Nocardia grow slowly, it usually takes $72 \mathrm{~h}$ to formed small colonies, so medical personnel should extend the time of bacteria culture to avoid the problems of misdiagnosis. But it is worth mentioning that small microflora could be seen which come from the positive blood culture and transferred to the blood plate for $24 \mathrm{~h}$, this may be the reason that nutrient solution in the blood cultures could neutralize the antibiotics or antibacterial factors which remaining in the specimen and makes bacteria grow quickly. It is timeconsuming and difficult for the traditional microbial identification method to identify the species of Nocardia, which can't meet the needs of the accurate and rapid clinical identification of pathogenic bacteria. But mass spectrometry, other modern molecular biology technologies, especially PCR and gene sequencing could provide a good foundation for rapid identification of Nocardia species, a recent report have shown that $91 \%$ of the Nocardia species could be accurate identified by the mass spectrometry system VITEK MS ${ }^{[12]}$. In this case reported here, the species identification results are different which were detected by mass spectrometry and 16S rRNA gene sequencing, this may because the species Nocardia vulneris were not in the library of the mass spectrometry system Bruker (Bruker Company, Bruker MALDI Biotyper TOF, America ) and VITEK MS (BioMerieux Company, BioMerieux VITEK MS, China), so the organisms was identified as Nocardia brasiliensis by Bruker and VITEK MS. This report suggests that $16 \mathrm{~S}$ rRNA gene sequencing method should be adopted, and the library of the mass spectrometry system should be timely upgrade or combined the results of gene sequencing.

Sulfonamides has been considered the first line of drug treatment of nocardiosis, a report regarding the antibiotic susceptibility of Nocardia showed that the resistance rate of Nocardia against sulfonamides is $57 \%$, and the mortality rate is higher when it used alone ${ }^{[13]}$. For immunosuppressive patients or the patients with disseminated infections, Trimethoprim-sulfamethoxazole monotherapy has been changed to Amikacin combined with Imipenem or Linezolid, which is for central nervous system infections or multidrug-resistant bacterial infections ${ }^{[14-16]}$. In this case, as the results that suspected Nocardia of the direct blood culture smear was received, the patient was treated by Trimethoprim-sulfamethoxazole combined with Meropenem for 3 days. Given the patient was still with a high fever, the treatment was changed to Trimethoprim-sulfamethoxazole combined with ceftriaxone for 2 days, but the patient's infection symptoms haven't prominently resolved, then following Trimethoprim-sulfamethoxazole combined with Linezolid for 1 day, which haven't showed efficacy, with a view to the isolate's drug 
sensitivity, the MIC values of Linezolid, Amikacin and Trimethoprim-sulfamethoxazole were far from the corresponding sensitive fold points, and as the patient was seriously ill, we considered treatment using Linezolid, Amikacin and Trimethoprim-sulfamethoxazole, at the same time, considering the patient with very poor renal function, the clinical pharmacists reduced the dosage of amikacin to prevent its damage in renal function, and the patient's temperature showed a decreasing trend after used trimethoprimsulfamethoxazole combination with linezolid and amikacin for 1 day, when treated with 15 days, the patient's infection symptoms had prominently resolved. Then the patient was treated with trimethoprimsulfamethoxazole combined with minocycline by oral agents for 6 months, and hadn't relapsed after cessation of the treatment. The successful treatment of this case fully demonstrates that the MIC value of the drug sensitivity test should be concerned when there is a wide choice of medicines, in addition, multidisciplinary cooperation may be warranted and it can effectively significantly improve the success rate of treatment. Scott et al. reported a case that in which retinitis caused by Nocardia veterana was

successfully treated by a combination of linezolid, amikacin, and meropenem ${ }^{[17]}$. For the cases which are diagnosed definitely should be treated with at least 12 months of antimicrobial therapy, bacteriological examination and antimicrobial susceptibility testing should be performed repeatedly. In addition, according to the antimicrobial sensitivity testing, the treatment regimen should be adjusted with the appropriate monitoring, and follow-ups are required to clear that whether the patient relapsed after cessation of the treatment.

\section{Conclusion}

In conclusion, this is the first reported case of disseminated infection of a macroglobulinemia patient caused by Nocardia vulneris. Despite the clinical characteristics, diagnostic methods and optimal treatment protocols for Nocardia vulneris remain unclear because of its rarity reported cases, our findings provides the information that $16 \mathrm{~S}$ rRNA gene sequencing method should be adopted or gene sequencing combined the results of mass spectrometry to diagnostic Nocardia vulneris infection. In addition, we suggest that the MIC value of the drug sensitivity test should be concerned when there is a wide choice of medicines, and for the disseminated cases which are diagnosed definitely should be treated with at least 12 months of antimicrobial therapy, bacteriological examination and antimicrobial susceptibility testing should be performed repeatedly.

\section{Declarations}

\section{Acknowledgement}

Funding: This work was supported by Natural Science Foundation of Fujian Province (Grant No. 2018J01408), Fujian provincial health technology project (Grant No. 2016-1-97).

\section{Conflict of interest statement}

The authors declare that they have no conflict of interest. 


\section{Ethics statement}

This study was approved by the Ethics Committee of Longyan First Affiliated Hospital of Fujian Medical University, Longyan, Fujian, China. The purpose and procedures of thestudy were carefully explained to all participants and written informed consent was obtained from all participants. All the clinical isolates analyzed in this work were collected as part of routine medical care. All the data analyzed in this work had already been anonymized before analysis.

\section{References}

1. Conville PS, Brown-Elliott BA, Smith T, Zelazny AM. The Complexities of Nocardia Taxonomy and Identification[J]. Journal of clinical microbiology. 2018;56(1).

2. Nakamura I, Nagakura T, Fujita H, Fukusima S, Gonoi T. Nocardia elegans infection: a case report and literature review[J]. International journal of infectious diseases : IJID : official publication of the International Society for Infectious Diseases. 2017;54:15-7.

3. Leli C, Moretti A, Guercini F, Cardaccia A, Furbetta L, Agnelli G, et al. Fatal Nocardia farcinica Bacteremia Diagnosed by Matrix-Assisted Laser Desorption-Ionization Time of Flight Mass Spectrometry in a Patient with Myelodysplastic Syndrome Treated with Corticosteroids[J]. Case reports in medicine. 2013;2013:368637.

4. Liu WL, Lai CC, Ko WC, Chen YH, Tang HJ, Huang YL, et al. Clinical and microbiological characteristics of infections caused by various Nocardia species in Taiwan: a multicenter study from 1998 to 2010[J]. European journal of clinical microbiology \& infectious diseases : official publication of the European Society of Clinical Microbiology. 2011;30(11):1341-7.

5. Martínez R, Reyes S, Menéndez R. Pulmonary nocardiosis: risk factors, clinical features, diagnosis and prognosis[J]. Current opinion in pulmonary medicine. 2008;14(3):219-27.

6. Smego RA, Jr., Gallis HA. The clinical spectrum of Nocardia brasiliensis infection in the United States[J]. Reviews of infectious diseases. 1984;6(2):164-80.

7. Paredes BE, Hunger RE, Braathen LR, Brand CU. Cutaneous nocardiosis caused by Nocardia brasiliensis after an insect bite[J]. Dermatology (Basel, Switzerland). 1999;198(2):159-61.

8. Koll BS, Brown AE, Kiehn TE, Armstrong D. Disseminated Nocardia brasiliensis infection with septic arthritis[J]. Clinical infectious diseases : an official publication of the Infectious Diseases Society of America. 1992;15(3):469-72.

9. Valdezate S, Garrido N, Carrasco G, Medina-Pascual MJ, Villalón P, Navarro AM, et al. Epidemiology and susceptibility to antimicrobial agents of the main Nocardia species in Spain[J]. The Journal of antimicrobial chemotherapy. 2017;72(3):754-61.

10. Coussement J, Lebeaux D, van Delden C, Guillot H, Freund R, Marbus S, et al. Nocardia Infection in Solid Organ Transplant Recipients: A Multicenter European Case-control Study[J]. Clinical infectious diseases : an official publication of the Infectious Diseases Society of America. 2016;63(3):338-45. 
11. Kontoyiannis DP, Ruoff K, Hooper DC. Nocardia bacteremia. Report of 4 cases and review of the literature[J]. Medicine. 1998;77(4):255-67.

12. Girard V, Mailler S, Polsinelli S, Jacob D, Saccomani MC, Celliere B, et al. Routine identification of Nocardia species by MALDI-TOF mass spectrometry[J]. Diagnostic microbiology and infectious disease. 2017;87(1):7-10.

13. Mootsikapun P, Intarapoka B, Liawnoraset W. Nocardiosis in Srinagarind Hospital, Thailand: review of 70 cases from 1996-2001[J]. International journal of infectious diseases : IJID : official publication of the International Society for Infectious Diseases. 2005;9(3):154-8.

14. Tremblay J, Thibert L, Alarie I, Valiquette L, Pépin J. Nocardiosis in Quebec, Canada, 1988-2008[J]. Clinical microbiology and infection : the official publication of the European Society of Clinical Microbiology and Infectious Diseases. 2011;17(5):690-6.

15. Welsh O, Vera-Cabrera L, Salinas-Carmona MC. Current treatment for nocardia infections[J]. Expert opinion on pharmacotherapy. 2013;14(17):2387-98.

16. Rivero A, García-Lázaro M, Pérez-Camacho I, Natera C, del Carmen Almodovar M, Camacho A, et al. Successful long-term treatment with linezolid for disseminated infection with multiresistant Nocardia farcinica[J]. Infection. 2008;36(4):389-91.

17. Scott M, Mehta S, Rahman HT, Grossniklaus HE, Yeh S. Nocardia veterana endogenous endophthalmitis in a cardiac transplant patient[J]. Journal of ophthalmic inflammation and infection. 2013;3(1):44.

\section{Figures}
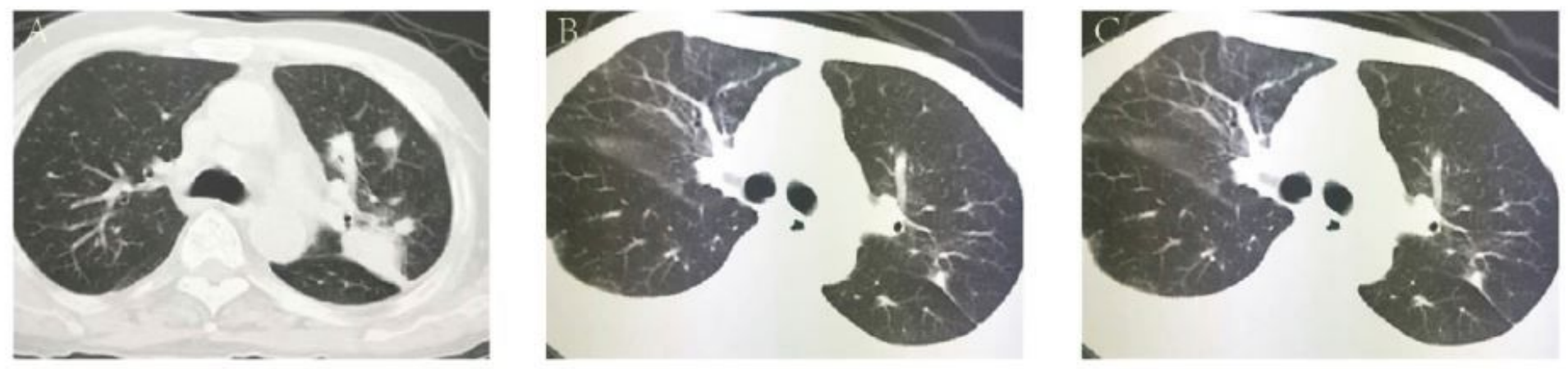

\section{Figure 1}

Pulmonary CT changes December 5, 2018 (A), January 22, 2019 (B), April 23, 2019 (C) 

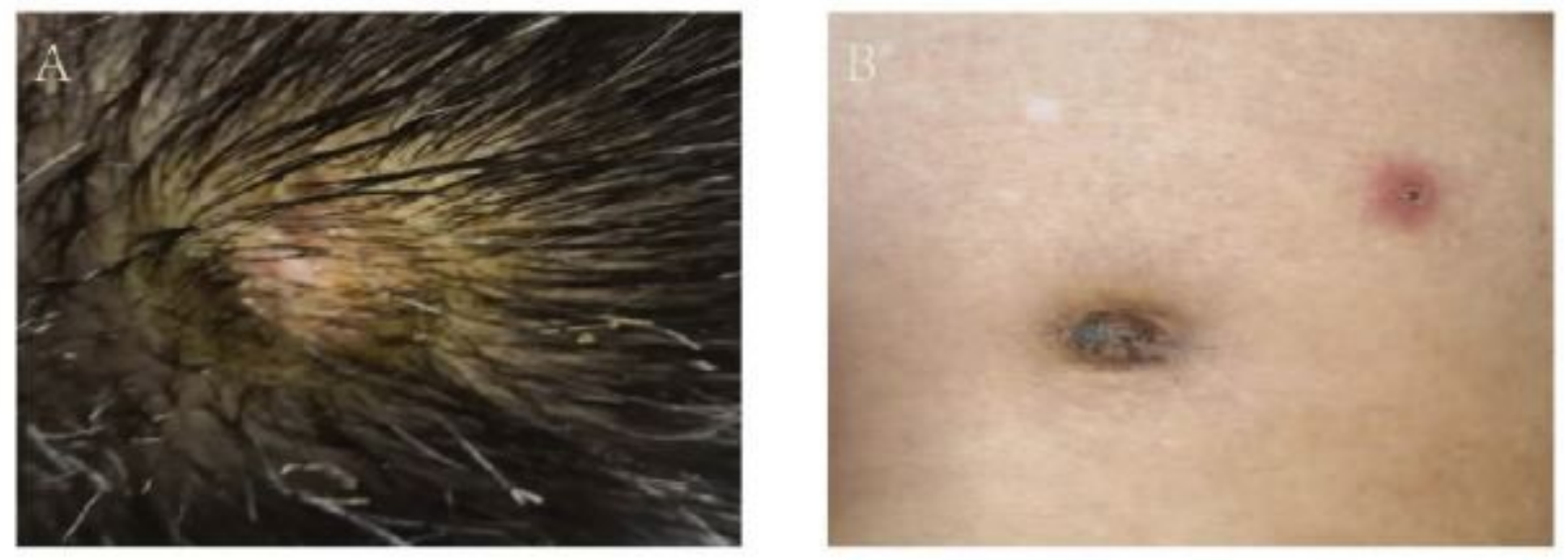

\section{Figure 2}

The rash on the patient's head $(A)$ and body $(B)$
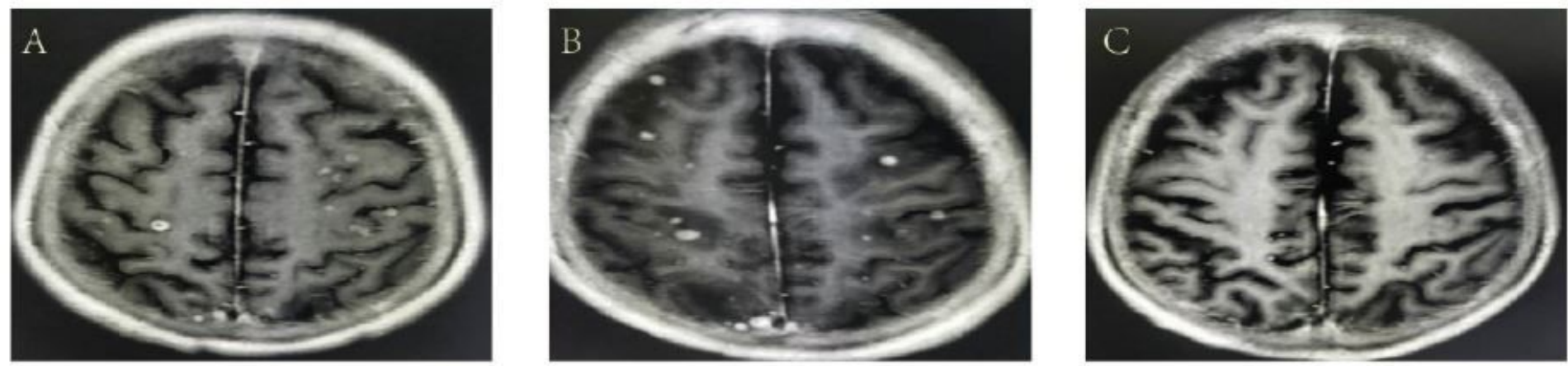

\section{Figure 3}

MRI changes in the brain during treatment Dec. 22, 2018 (A), Jan. 17, 2019 (B), May 24, 2019 (C)
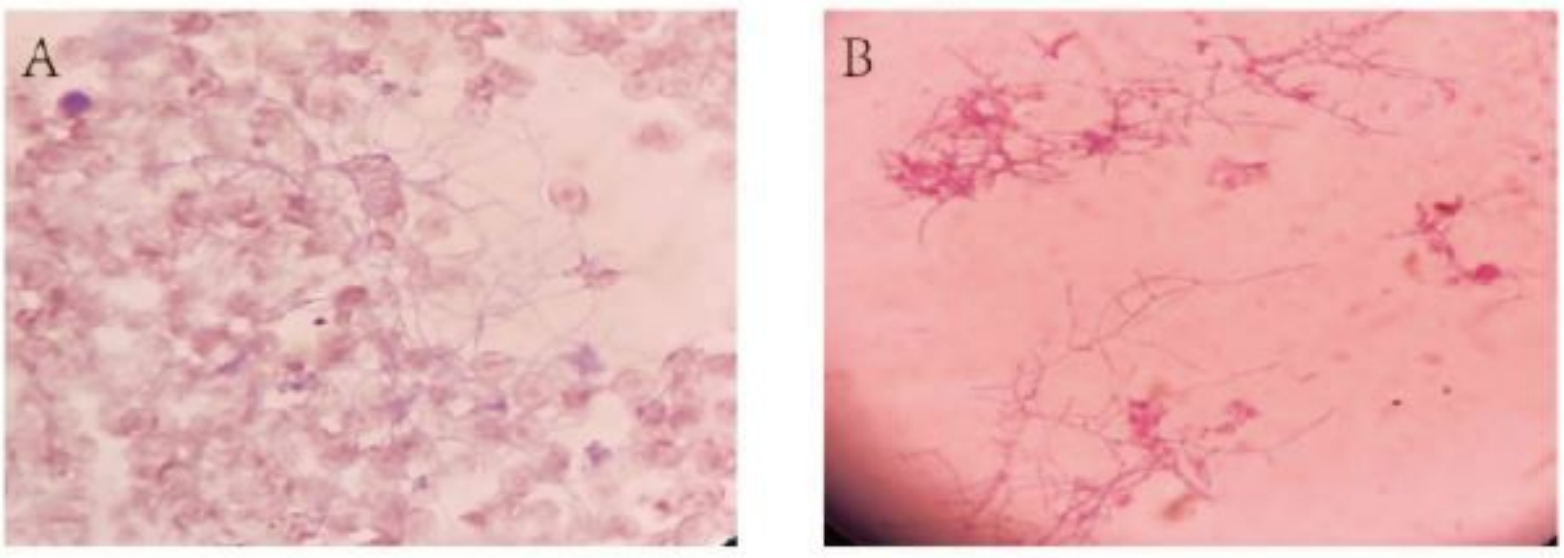

\section{Figure 4}

Direct blood culture smear reigh's staining (A) gram staining (B) (X 1000) 

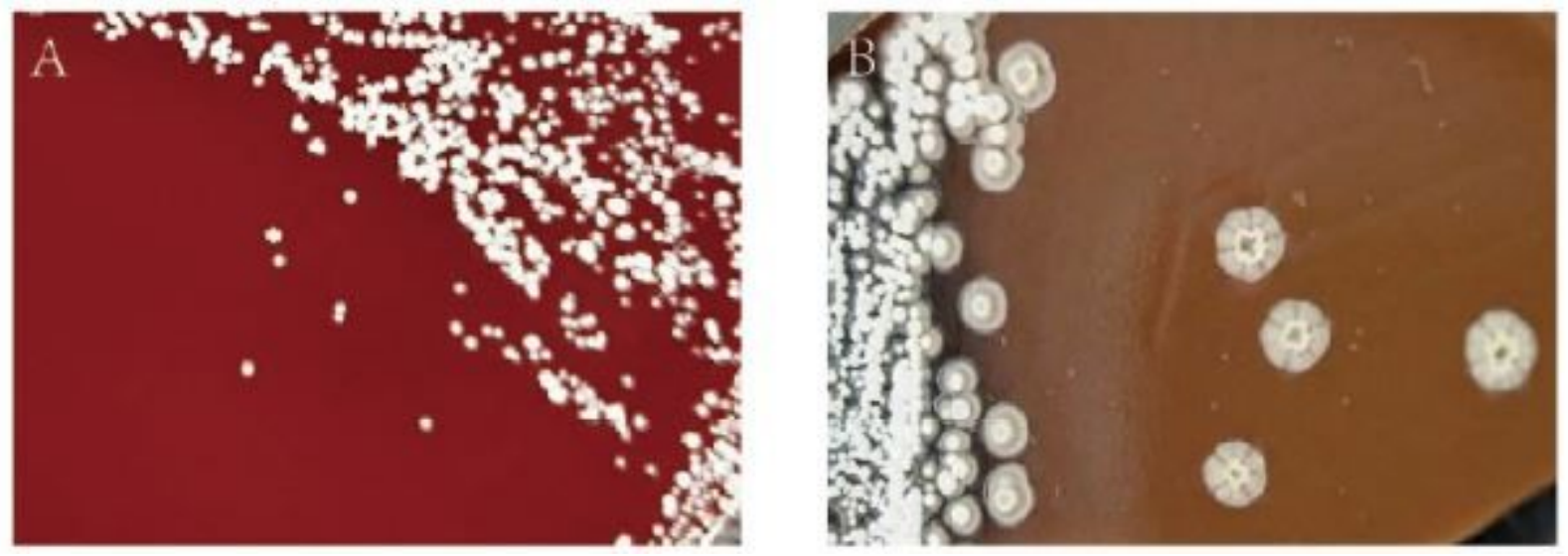

Figure 5

Colonies of Nocardia vulneris cultured for 3 days and 15 days
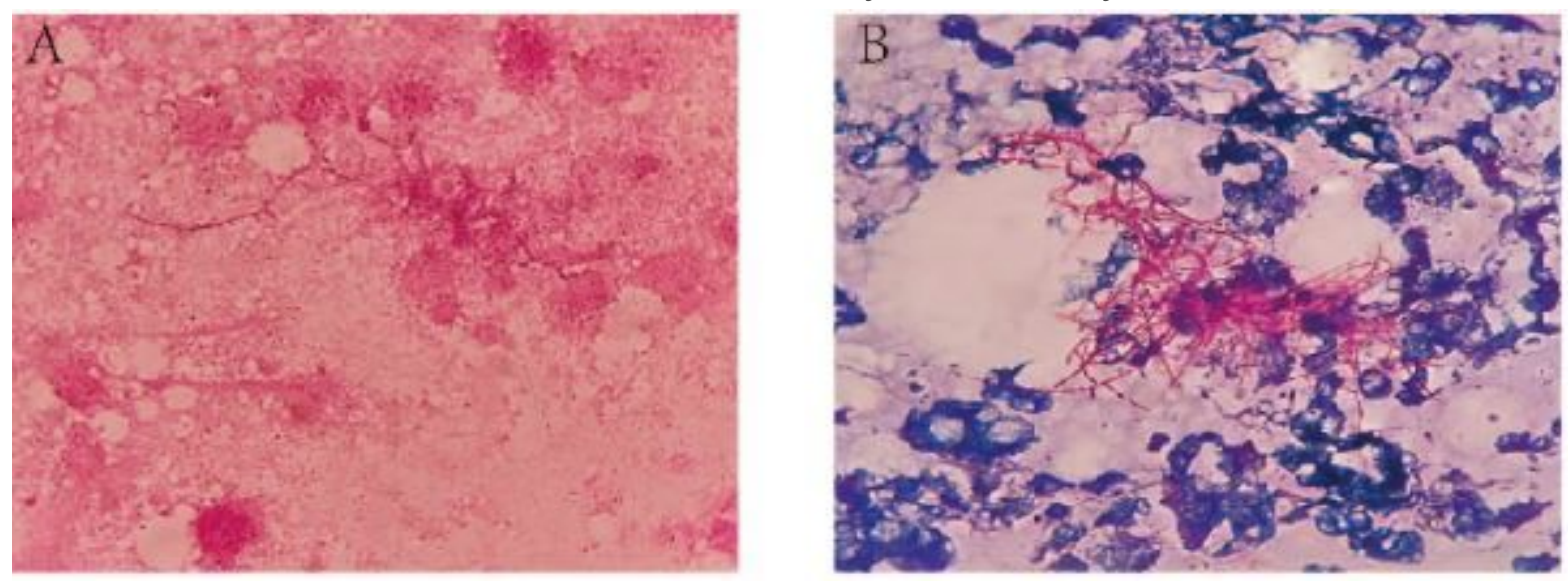

Figure 6

Gram staining (A) and weak acid-fast staining (B)

\section{Supplementary Files}

This is a list of supplementary files associated with this preprint. Click to download.

- Authorsinformation.pdf

- coverletter.pdf 